\title{
GRAPH RELATIONS, CLIQUE DIVERGENCE AND SURFACE TRIANGULATIONS.
}

\author{
F. LARRIÓN, V. NEUMANN-LARA, AND M.A. PIZAÑA
}

\begin{abstract}
This work has two aims: First, we introduce a powerful technique for proving clique divergence when the graph satisfies a certain symmetry condition. Second, we prove that each closed surface admits a clique divergent triangulation. By definition, a graph is clique divergent if the orders of its iterated clique graphs tend to infinity, and the clique graph of a graph is the intersection graph of its maximal complete subgraphs.
\end{abstract}

\section{INTRODUCTION}

All our graphs are simple, non-empty, finite and connected. We identify induced subgraphs and vertex-sets. The clique graph $K(G)$ of a graph $G$ is the intersection graph of the family of all its cliques (maximal complete subgraphs). The iterated clique graphs $K^{n}(G)$ are given by $K^{n+1}(G)=K\left(K^{n}(G)\right)$. We refer to $[12,5,15]$ for the literature on iterated clique graphs. We study the dynamical behaviour of graphs under the iterates of the clique operator $K$. There are two main types of $K$-behaviour: $G$ is clique convergent if $K^{n}(G) \cong K^{m}(G)$ for some pair $n<m$; in other words, $G$ is clique convergent iff $G$ is, in the obvious sense, eventually clique periodic. On the other hand, $G$ is clique divergent if $\left|V\left(K^{n}(G)\right)\right|$ tends to infinity with $n$ or, equivalently, if this sequence of orders is unbounded. With this terminology, a graph is clique divergent iff it is not clique convergent.

To prove directly that a graph is clique divergent one all but needs to know explicitly all its iterated clique graphs. This has only been possible for a handful of cases: octahedra [9], locally $C_{6}$ graphs [4] and clockwork graphs [5]. Indirect methods are scarce but more fruitful: For instance, any graph with a clique divergent retract is clique divergent [9]. Also, in any finite triangular covering map [4], the cover and the base have the same $K$-behaviour (see 4.2 below). Another indirect approach is to prove something stronger than clique divergence: expansivity in [10], rank divergence in this work.

The method developed in $\S 2$ can be sketched as follows: A coaffine graph has a fixed symmetry: an automorphism $\sigma$ which sends each vertex far away from it. The rank of such a graph is the maximum possible number of $\sigma$-invariant subgraphs which are mutually disjoint but close to each other. If the rank of $K^{n}(G)$ tends to infinity with $n$, then $G$ is rank divergent and hence clique divergent. A graph relation from one graph to another is a relation between their vertex-sets such that the image of any complete subgraph is complete. Then we have Theorem 2.6: Given a symmetry-preserving graph relation between coaffine graphs, if the domain is rank divergent then so is the codomain. Our $§ 2$ generalizes, strengthens and simplifies some of the results outlined mostly without proofs in $[9,10]$. The major new features are the use of graph relations, the coaffinity indexes and the 
notion of rank divergence. It is noteworthy that in this context it is easier to work with graph relations than with homomorphisms of graphs.

Iterated clique graphs are particularly interesting for graphs that triangulate some closed surface. More restrictively, a Whitney triangulation [6] is an embedding of a graph in a closed surface in such a way that the faces of the embedding are precisely the cliques (necessarily triangles) of the graph; in other words, each face is a triangle, each triangle is a face and the graph is not $K_{4}$. Equivalently, one has a simplicial complex $\mathbb{k}$, the geometric realization $|\mathbb{k}|$ is the surface, the graph is the 1 -skeleton $\mathbb{k}_{1}$ and each clique of the graph is a (maximal) simplex of $\mathbb{k}$. Whitney triangulations abundantly exist: the barycentric subdivision of any surface triangulation is Whitney. For these triangulations, the complex is uniquely determined by the graph, so we use the same name for the graph and the complex. Call a graph locally cyclic if the open neighborhood of each vertex is a cycle. Then the graph $G$ defines some Whitney triangulation if and only if $G$ is locally cyclic and its minimum degree satisfies $\delta(G)>3$ (but note that the only locally cyclic $G$ with $\delta(G) \leq 3$ is $K_{4}$ ). With other names, Whitney triangulations have been studied also by Tutte in [13] and by Hartsfield and Ringel in [3]. In 1931, Whitney [14] proved that any Whitney triangulation of the sphere is Hamiltonian.

The $K$-behaviour of regular Whitney triangulations is known and completely determined by the topology: If the Whitney triangulation $G$ is a regular graph, then it is clique convergent if and only if its Euler characteristic is negative. If $G$ is $r$-regular, this simply means that $r \geq 7$. Despite its simple statement, this result for the regular case took a long time in coming and its whole proof is contained in 5 papers, see [6].

In the non-regular case the $K$-behaviour is not completely determined by the topology: On the one hand, it is known [7] that all closed surfaces with negative Euler characteristic admit a clique convergent Whitney triangulation, and even [6] that all Whitney triangulations with $\delta(G) \geq 7$ are clique convergent. On the other hand, we shall prove in $\S 4$ that each closed surface admits infinitely many clique divergent Whitney triangulations (see Theorem 4.3). However, as will be seen in $\S 5$, we conjecture that at least in some non-regular cases the topology still does determine the $K$-behaviour.

Theorem 4.3 is a strong result which will illustrate the power of our methods in $\S 2$, but in order to apply Theorem 2.6 to the proof of Theorem 4.3 we will need to have some rank divergent graphs. These shall be constructed in $§ 3$ using results on clockwork graphs from [5].

\section{Relations, Coaffinity and Rank Divergent Graphs}

If $G$ is a graph, for a vertex $x \in V(G)$ we usually write just $x \in G$. If $x, y \in G$, we denote the distance from $x$ to $y$ by $d_{G}(x, y)$ or just $d(x, y)$; also, we will write $x \simeq y$ when $x$ and $y$ are either adjacent or equal, i.e. when $d(x, y) \leq 1$.

Recall that, for sets $A$ and $B$, a relation $f: A \rightarrow B$ is any subset $f$ of the cartesian product $A \times B$. A relation $f$ is known once we know the sets $A$ and $B$ and, for all $a \in A$, the image set $f(a)=\{b \in B:(a, b) \in f\}$. If $f(a)=\{b\}$ we can also write $f(a)=b$ so as to regain the usual notation when $f$ is a function. The image of $X \subseteq A$ is, of course, $f(X)=\bigcup_{x \in X} f(x)$. If $f: A \rightarrow B$ and $g: B \rightarrow C$ are relations, their composite $g \circ f: A \rightarrow C$ is given by $[g \circ f](a)=g(f(a))$. 
For graphs $A$ and $B$, a graph relation $f: A \rightarrow B$ is a relation $f: V(A) \rightarrow V(B)$ where $f(X)$ is complete in $B$ whenever $X$ is so in $A$. In other words, we have that $f(a) \neq \varnothing$ for all $a \in A$ and also $a \simeq a^{\prime}$ implies $b \simeq b^{\prime}$ for all $b \in f(a)$ and $b^{\prime} \in f\left(a^{\prime}\right)$. Any composite of graph relations is a graph relation. It is also clear that ordinary morphisms of graphs are graph relations. Note that if $\left(x_{1}, \ldots, x_{s}\right)$ is a path in $A$ and $y_{i} \in f\left(x_{i}\right)$, then $\left(y_{1}, \ldots, y_{s}\right)$ is a walk in $B$, so we have:

Lemma 2.1. Let $f: A \rightarrow B$ be a graph relation, and $a, a^{\prime} \in A, a \neq a^{\prime}$. Then, if $b \in f(a)$ and $b^{\prime} \in f\left(a^{\prime}\right)$, we have $d_{B}\left(b, b^{\prime}\right) \leq d_{A}\left(a, a^{\prime}\right)$.

Any graph relation $f: A \rightarrow B$ induces a graph relation $f_{K}: K(A) \rightarrow K(B)$ given by $f_{K}(Q)=\{P \in K(B): f(Q) \subseteq P\}$ for each $Q \in K(A)$. This $f_{K}$ is indeed a graph relation: If $Q \simeq Q^{\prime}$ in $K(A)$, take $P \in f_{K}(Q)$ and $P^{\prime} \in f_{K}\left(Q^{\prime}\right)$; then $P \cap P^{\prime} \supseteq f(Q) \cap f\left(Q^{\prime}\right) \supseteq f\left(Q \cap Q^{\prime}\right) \neq \varnothing$, so $P \simeq P^{\prime}$ in $K(B)$.

In general $f_{K}$ needs not to be a function even if $f$ is so but, when $f: A \rightarrow B$ is an isomorphism, then $f_{K}: K(A) \rightarrow K(B)$ is also an isomorphism: Indeed, $f(Q)$ is already a clique of $B$ whenever $Q$ is a clique of $A$, so $f_{K}$ is univalued. In a similar way, if $f: A \rightarrow B$ is a local isomorphism then $f_{K}: K(A) \rightarrow K(B)$ is also a local isomorphism (see 4.2 below).

An automorphic graph $\mathbb{A}$ is a pair $\mathbb{A}=(A, \alpha)$ where $A$ is a graph and $\alpha$ is an automorphism of $A$. Given two automorphic graphs $\mathbb{A}=(A, \alpha)$ and $\mathbb{B}=(B, \beta)$, an admissible relation $f: \mathbb{A} \rightarrow \mathbb{B}$ is a graph relation $f: A \rightarrow B$ satisfying $f \circ \alpha=\beta \circ f$. In other words, an admissible relation $f: \mathbb{A} \rightarrow \mathbb{B}$ is just a symmetry-preserving graph relation. Any composite of admissible relations is also admissible.

For any automorphic graph $\mathbb{A}=(A, \alpha)$ we define $K(\mathbb{A})=\left(K(A), \alpha_{K}\right)$. As previously noted, $\alpha_{K}$ is an automorphism, so $K(\mathbb{A})$ is also automorphic.

Proposition 2.2. Let $f: \mathbb{A} \rightarrow \mathbb{B}$ be an admissible relation between automorphic graphs. Then $f_{K}: K(\mathbb{A}) \rightarrow K(\mathbb{B})$ is also an admissible relation.

Proof. Let $\mathbb{A}=(A, \alpha), \mathbb{B}=(B, \beta)$. We already know that $f_{K}: K(A) \rightarrow K(B)$ is a graph relation. We prove that $f_{K}$ is admissible. If $Q \in K(A)$, we have:

$$
\begin{aligned}
f_{K}\left(\alpha_{K}(Q)\right) & =f_{K}(\alpha(Q))=\left\{P^{\prime} \in K(B): f(\alpha(Q)) \subseteq P^{\prime}\right\} \\
& =\left\{P^{\prime} \in K(B): \beta(f(Q)) \subseteq P^{\prime}\right\} \\
& =\{\beta(P) \in K(B): \beta(f(Q)) \subseteq \beta(P)\} \\
& =\{\beta(P) \in K(B): f(Q) \subseteq P\} \\
& =\beta_{K}(\{P \in K(B): f(Q) \subseteq P\}) \\
& =\beta_{K}\left(f_{K}(Q)\right) .
\end{aligned}
$$

Therefore, $f_{K} \circ \alpha_{K}=\beta_{K} \circ f_{K}$ and thus $f_{K}$ is admissible.

Let $\mathbb{A}=(A, \alpha)$ be automorphic and $r \geq 2$ an integer. We say that $\mathbb{A}$ is $r$ coaffine (and that $\alpha$ is an $r$-coaffination) if $d(x, \alpha(x)) \geq r$ for every $x \in A$. In order to show that $K(\mathbb{A})$ is also $r$-coaffine we need to calculate distances in $K(G)$. If $Q, Q^{\prime} \in K(G)$, denote by $\mathcal{D}_{G}\left(Q, Q^{\prime}\right)$ the set $\left\{d_{G}\left(x, x^{\prime}\right): x \in Q, x^{\prime} \in Q^{\prime}\right\}$. The following result from [11] is quite useful:

Proposition 2.3. Let $G$ be a graph and $Q, Q^{\prime} \in K(G)$ with $Q \neq Q^{\prime}$. Then:

$$
d_{K(G)}\left(Q, Q^{\prime}\right)=\min \mathcal{D}_{G}\left(Q, Q^{\prime}\right)+1 \text {. }
$$

Proposition 2.4. If $\mathbb{A}=(A, \alpha)$ is an r-coaffine graph then so is $K(\mathbb{A})$. 
Proof. We know already that $K(\mathbb{A})$ is automorphic. Let $Q \in K(A)$ and let $x \in Q$ and $y \in \alpha_{K}(Q)$ be such that $d_{A}(x, y)=\min \mathcal{D}_{A}\left(Q, \alpha_{K}(Q)\right)$. Since $\alpha(x) \simeq y$ and $d(x, \alpha(x)) \geq r$, we have $d(x, y) \geq r-1$. Since $r \geq 2, Q \neq \alpha_{K}(Q)$. By 2.3 we get that $d_{K(A)}\left(Q, \alpha_{K}(Q)\right) \geq r$, so $K(\mathbb{A})$ is $r$-coaffine.

Fix $r \geq 2$ and let $\mathbb{A}=(A, \alpha)$ be $r$-coaffine. The $\operatorname{rank}$ of $\mathbb{A}$ is the greatest $n$ such that there exist non-empty, pairwise disjoint, $\alpha$-invariant sets $A_{1}, \ldots, A_{n} \subseteq V(A)$ such that $d_{A}\left(a_{i}, a_{j}\right)<r$ whenever $a_{i} \in A_{i}, a_{j} \in A_{j}$ and $i \neq j$. The same concept is obtained if we ask the sets $A_{i}$ to be $\alpha$-orbits.

Theorem 2.5. Let $f: \mathbb{A} \rightarrow \mathbb{B}$ be an admissible relation between $r$-coaffine graphs. Then $\operatorname{rank}(\mathbb{A}) \leq \operatorname{rank}(\mathbb{B})$.

Proof. Let $\mathbb{A}=(A, \alpha), \mathbb{B}=(B, \beta), \operatorname{rank}(\mathbb{A})=n$. Take $A_{1}, \ldots, A_{n} \subseteq V(A)$ as above. Since $\beta\left(f\left(A_{i}\right)\right)=f\left(\alpha\left(A_{i}\right)\right)=f\left(A_{i}\right), f\left(A_{i}\right)$ is $\beta$-invariant. If $f\left(A_{i}\right) \cap f\left(A_{j}\right) \neq$ $\varnothing$ with $i \neq j$, take $z, \beta(z) \in f\left(A_{i}\right) \cap f\left(A_{j}\right)$ and $x \in A_{i}, y \in A_{j}$ with $z \in f(x)$, $\beta(z) \in f(y)$. By $2.1, d_{B}(z, \beta(z)) \leq d_{A}(x, y)<r$, contradicting that $\mathbb{B}$ is $r$-coaffine. Also by 2.1 , we have that $d_{B}\left(z_{i}, z_{j}\right)<r$ for every $z_{i} \in f\left(A_{i}\right), z_{j} \in f\left(A_{j}\right), i \neq j$. Since the $f\left(A_{i}\right)$ are non-empty, they satisfy the definition of rank (save perhaps for the maximality of $n$ ) so $\operatorname{rank}(\mathbb{A})=n \leq \operatorname{rank}(\mathbb{B})$.

Let $\mathbb{A}=(A, \alpha)$ be $r$-coaffine. We say that $\mathbb{A}$ is rank divergent if the sequence $\left\{\operatorname{rank}\left(K^{n}(\mathbb{A})\right)\right\}_{n=1,2, \ldots}$ is not bounded. Since $\operatorname{rank}(\mathbb{A})<|V(A)|$, it follows that every rank divergent graph is clique divergent. Note that if some $K^{n}(\mathbb{A})$ is rank divergent, then $\mathbb{A}$ itself is rank divergent.

Theorem 2.6. Let $f: \mathbb{A} \rightarrow \mathbb{B}$ be an admissible relation between $r$-coaffine graphs. Then, if $\mathbb{A}$ is rank divergent, so is $\mathbb{B}$.

Proof. This follows using iteratively 2.2, 2.4 and 2.5 .

\section{Examples of Rank Divergent Graphs}

In order to use Theorem 2.6, one needs to know some rank divergent graph. Here we construct some examples. Using the theory of clockwork graphs [5], we will be able to describe explicitly all their iterated clique graphs. In fact, our examples constitute the simplest and more symmetric kind of clockwork graphs.

For $m \geq 2$ and $n \geq 0$, we shall define a graph $R_{2 m}^{n}$. The integer $2 m$ is called the number of segments, and it is convenient to consider it fixed while $n$ varies. Fig. 1 depicts $R_{2 m}^{n}$ for $m=2$ and $n=0,1,2$. For clarity, some vertices (white dots) and some edges (dashed lines) have been drawn twice. These drawing conventions are also used in the other figures of this paper.

Our clockwork graph $R_{2 m}^{n}$ is composed of two parts: the crown and the core. The crown is a cylindrical ladder with $2 m$ squares; it is induced by the vertices with lower indices 0 and 1 . This crown is common to all the graphs $R_{2 m}^{n}$ with the same fixed $m$. The complement of the crown is the core, which is the $n$-th power of the cycle with $2 m(n+1)$ vertices; in Fig. 1 it is induced by the vertices with lower indices from 2 to $n+2$. The vertices of the core are partitioned into $2 m$ segments of $n+1$ consecutive vertices each, and each segment lies inside of a square of the crown, in the sense that each vertex of the segment is a neighbour of the four vertices of the square. A more concise description is the following:

For $m \geq 2$ and $n \geq 0$ define the graph $R_{2 m}^{n}$ (see Fig. 1) by: 

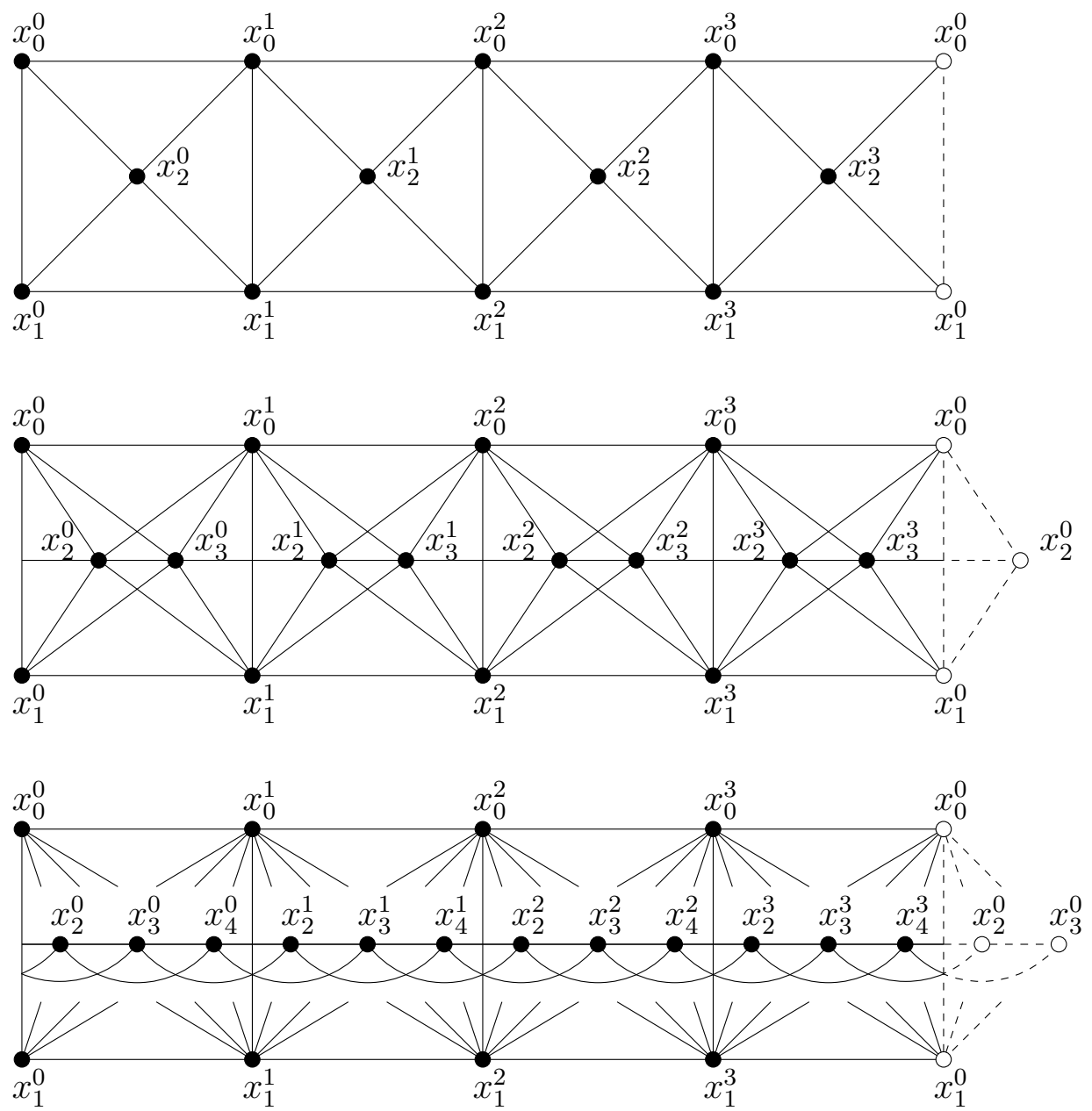

Figure 1. From top to bottom: $R_{4}^{0}, R_{4}^{1}$ and $R_{4}^{2}$

$$
\begin{gathered}
V\left(R_{2 m}^{n}\right)=\left\{x_{l}^{u}: u \in \mathbb{Z}_{2 m} \text { and } l \in\{0,1, \ldots, n+2\}\right\} \text { and } \\
\left\{x_{l}^{u}, x_{l^{\prime}}^{u^{\prime}}\right\} \in E\left(R_{2 m}^{n}\right) \text { if }\left\{\begin{array}{l}
u=u^{\prime} \text { and } l \neq l^{\prime}, \text { or } \\
u^{\prime}=u+1, l \in\{0,1\} \text { and } l^{\prime}=l \text {, or } \\
u^{\prime}=u+1, l \notin\{0,1\} \text { and } l^{\prime}<l .
\end{array}\right.
\end{gathered}
$$

It follows from [5] (see also the short version in [8]) that there is an isomorphism $\psi_{n}: K\left(R_{2 m}^{n}\right) \longrightarrow R_{2 m}^{n+1}$, but for the reader's convenience we shall explicitly describe the cliques of $R_{2 m}^{n}$ and the isomorphism $\psi_{n}$. For each vertex $v=x_{l}^{u} \in R_{2 m}^{n}$ there is a clique $Q_{l}^{u}$ which starts at this vertex: If $l \in\{0,1\}$, the vertex $v$ lies in the crown and the clique contains the crown's edge $e=\left\{x_{l}^{u}, x_{l}^{u+1}\right\}$ starting at $v$ and the core's segment in the square containing $e$, that is, $Q_{l}^{u}=$ $\left\{x_{l}^{u}, x_{l}^{u+1}, x_{2}^{u}, x_{3}^{u}, \ldots, x_{n+2}^{u}\right\}$. If $l \in\{2, \ldots, n+2\}$, the vertex $v$ lies in the core, and the clique contains the step of the ladder that lies ahead and the core's clique starting at $v: Q_{l}^{u}=\left\{x_{0}^{u+1}, x_{1}^{u+1}, x_{l}^{u}, \ldots, x_{n+2}^{u}, x_{2}^{u+1}, \ldots, x_{l-1}^{u+1}\right\}$. We also have, for each step $\left\{x_{0}^{u}, x_{1}^{u}\right\}$ of the ladder, a new clique $Q(u)$ containing that step and the next 
segment of the core: $Q(u)=\left\{x_{0}^{u}, x_{1}^{u}, x_{2}^{u}, \ldots, x_{n+2}^{u}\right\}$. If we rename the vertices of $R_{2 m}^{n+1}$ as $V\left(R_{2 m}^{n+1}\right)=\left\{y_{l}^{u}: u \in \mathbb{Z}_{2 m}\right.$ and $\left.l \in\{0,1, \ldots, n+3\}\right\}$, the isomorphism $\psi_{n}: K\left(R_{2 m}^{n}\right) \rightarrow R_{2 m}^{n+1}$ is given by $\psi_{n}\left(Q_{l}^{u}\right)=y_{l}^{u}$ and $\psi_{n}(Q(u))=y_{n+3}^{u-1}$.

The graph $R_{2 m}^{n}$ has a natural antipodal symmetry which sends everything $m$ segments ahead and then interchanges the two borders (lower indices 0 and 1 ) of the crown. In other words, we define the function $\rho_{2 m}^{n}: V\left(R_{2 m}^{n}\right) \rightarrow V\left(R_{2 m}^{n}\right)$ by:

$$
\rho_{2 m}^{n}\left(x_{l}^{u}\right)=\left\{\begin{array}{l}
x_{1}^{u+m} \text { if } l=0 \\
x_{0}^{u+m} \text { if } l=1 \\
x_{l}^{u+m} \text { otherwise. }
\end{array}\right.
$$

It is quite clear that $\rho_{2 m}^{n}$ is an automorphism of $R_{2 m}^{n}$, so we have an automorphic graph $\mathbb{R}_{2 m}^{n}=\left(R_{2 m}^{n}, \rho_{2 m}^{n}\right)$. It is also easy to see that $\psi_{n} \circ\left(\rho_{2 m}^{n}\right)_{K}=\rho_{2 m}^{n+1} \circ \psi_{n}$, so $\psi_{n}: K\left(\mathbb{R}_{2 m}^{n}\right) \rightarrow \mathbb{R}_{2 m}^{n+1}$ is an admissible isomorphism. We conclude that there is an admissible isomorphism $K^{s}\left(\mathbb{R}_{2 m}^{n}\right) \cong \mathbb{R}_{2 m}^{n+s}$ for each $s \geq 1$.

Theorem 3.1. $\mathbb{R}_{2 m}^{n}$ is an $(m+1)$-coaffine rank divergent graph.

Proof. Consider the segments $S_{u}=\left\{x_{0}^{u}, x_{1}^{u}, x_{2}^{u}\right\}$ of $R_{2 m}^{0}$ and note that in $R_{2 m}^{0}$ there are only two kind of edges: those joining vertices in the same $S_{u}$ and those joining vertices in consecutive segments $S_{u}$ and $S_{u+1}$. Since any shortest path between $x_{l}^{u}$ and $\rho_{2 m}^{0}\left(x_{l}^{u}\right)=x_{l^{\prime}}^{u+m}$ must pass through at least $m+1$ segments (including both ends) and must also use at least two vertices from at least one of these segments, it follows that $d\left(x_{l}^{u}, \rho_{2 m}^{0}\left(x_{l}^{u}\right)\right) \geq m+1$. Therefore $\mathbb{R}_{2 m}^{0}$ is $(m+1)$-coaffine. Using 2.4 repeatedly we get that $\mathbb{R}_{2 m}^{n} \cong K^{n}\left(\mathbb{R}_{2 m}^{0}\right)$ is $(m+1)$-coaffine for all $n$.

To prove rank divergence, let $A_{t}=\left\{x_{2+m t}^{0}, x_{2+m t}^{m}\right\}$ for $t=0,1, \ldots,\left\lfloor\frac{n}{m}\right\rfloor$. These sets are clearly non-empty, $\rho_{2 m}^{n}$-invariant, and pairwise disjoint. Now, assuming that $t^{\prime}<t$, the following explicit paths:

$$
\begin{aligned}
& \left(x_{2+m t}^{0}, x_{2+m t-1}^{1}, \ldots, x_{2+m(t-1)+1}^{m-1}, x_{2+m t^{\prime}}^{m}\right) \\
& \left(x_{2+m t}^{m}, x_{2+m t-1}^{m+1}, \ldots, x_{2+m(t-1)+1}^{2 m-1}, x_{2+m t^{\prime}}^{0}\right)
\end{aligned}
$$

show us that $d\left(a_{t}, a_{t^{\prime}}\right) \leq m$ for $a_{t} \in A_{t}, a_{t^{\prime}} \in A_{t^{\prime}}$ and $t \neq t^{\prime}$. Therefore, we have $\operatorname{rank}\left(\mathbb{R}_{2 m}^{n}\right) \geq\left\lfloor\frac{n}{m}\right\rfloor+1$. From $K^{s}\left(\mathbb{R}_{2 m}^{n}\right) \cong \mathbb{R}_{2 m}^{n+s}$, we get that $\mathbb{R}_{2 m}^{n}$ is rank divergent.

Theorems 2.6 and 3.1 immediately yield huge families of rank divergent graphs: just take any of these coaffine graphs $\mathbb{R}=(R, \rho)=\mathbb{R}_{2 m}^{n}$ and add any number of vertices and edges with the sole restriction of preserving the symmetry and the $(m+1)$-coaffinity. For instance, a very simple construction always works: Take any vertex $x \in \mathbb{R}$, attach some graph to it and then do exactly the same to $\rho(x)$; now extend $\rho$ in the obvious way. This can clearly be iterated.

Many more familiar examples can also be readily seen to be rank divergent: Among them, Fig. 2 depicts the stellated cube and the barycentric subdivision of the octahedron. Here by stellating we mean replacing faces by wheels. In both cases, an admissible subgraph isomorphic to some $\mathbb{R}_{2 m}^{0}$ is highlighted. The icosahedron and the stellated dodecahedron depicted in Fig. 3 are also rank divergent: This time we found an admissible embedding of some $\mathbb{R}_{2 m}^{0}$ into their clique graphs. In all these four examples the antipodal map was used as coaffination.

A more systematic approach will presently yield the abundant existence of clique divergent triangulations for all surfaces. 

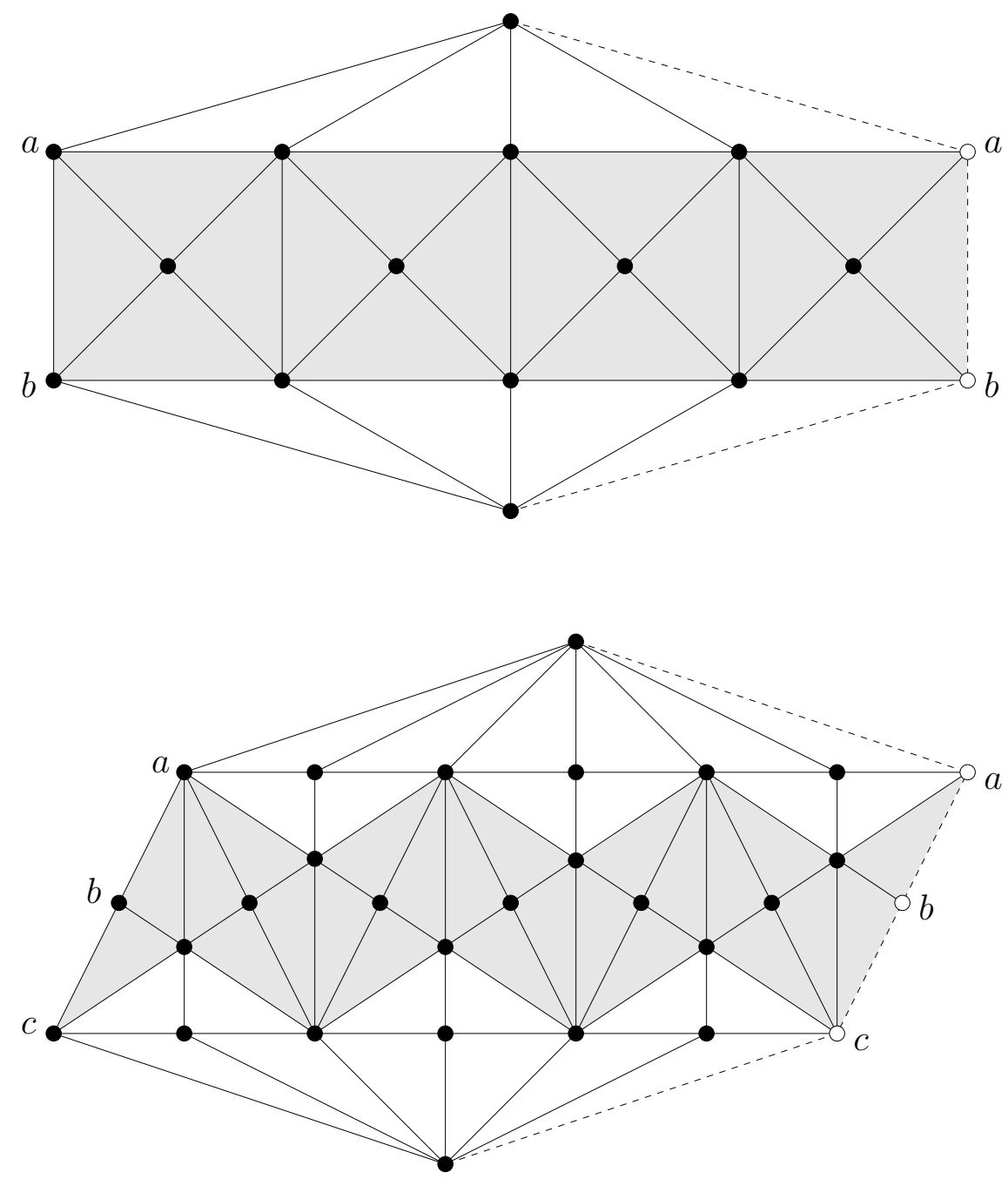

Figure 2. Stellated cube and barycentric subdivision of the octahedron.

\section{Clique Divergent Surface Triangulations}

For auxiliary purposes, we will need to speak also of Whitney triangulations of compact surfaces, not only of closed surfaces. It is clear that the graphs involved in this more general setting are such that each open neighbourhood is either a cycle of length at least 4 or a non-trivial path.

Theorem 4.1. Let $\mathcal{S}$ be an orientable closed surface and fix an integer $r \geq 3$. Then there are infinitely many Whitney triangulations $T$ of $\mathcal{S}$ admitting an automorphism $\sigma: T \rightarrow T$ such that:

1. $\sigma^{2}=1_{T}$,

2. $\sigma$ is orientation-reversing and

3. $\mathbb{T}=(T, \sigma)$ is $r$-coaffine rank divergent. 

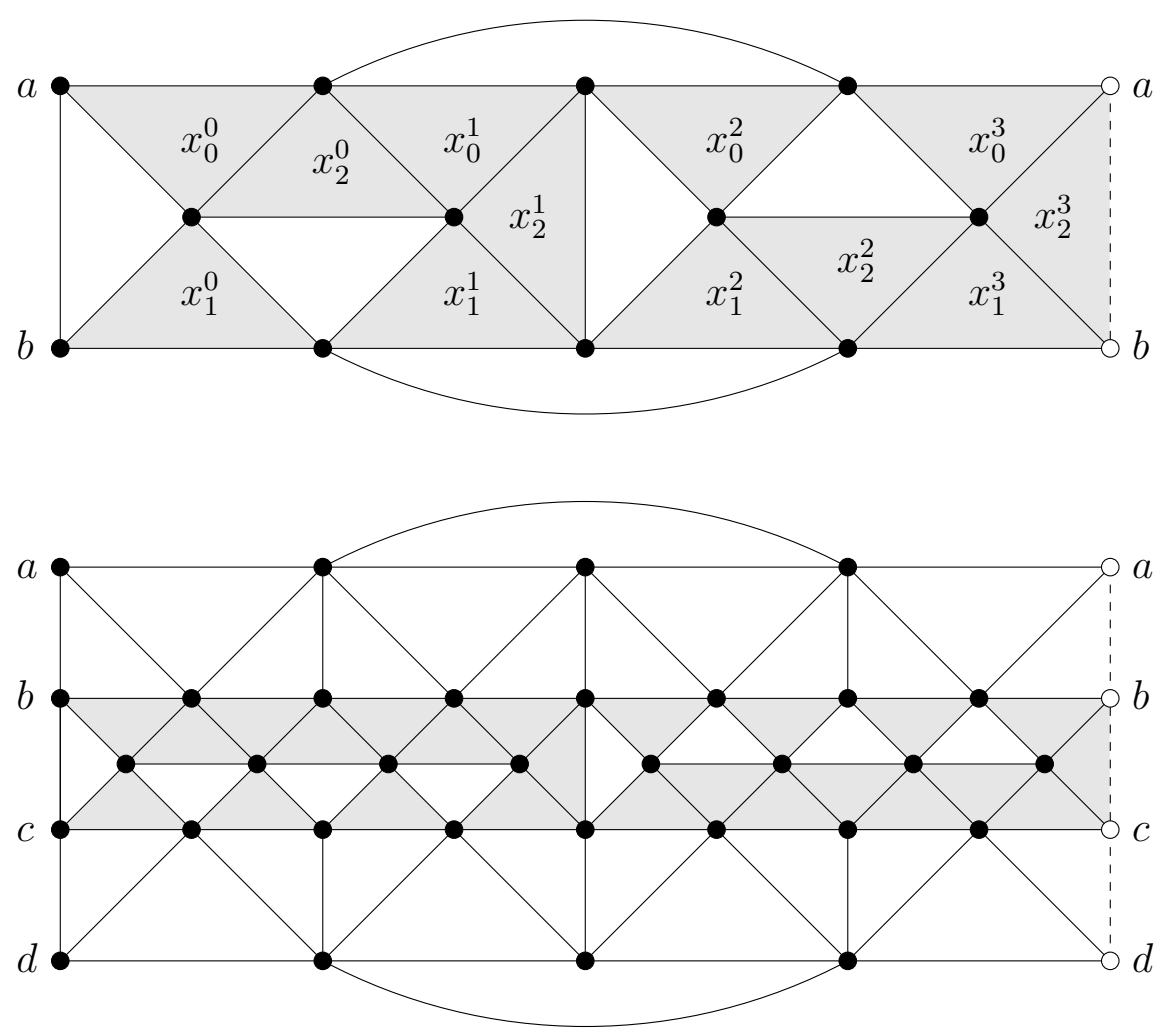

Figure 3. Icosahedron and stellated dodecahedron.

Proof. We proceed by induction on the genus $g$ of $\mathcal{S}$. Start with the Whitney triangulation $\mathbb{R}=\mathbb{R}_{2(r-1)}^{0}$ of the cylinder. There are many ways to extend it to a triangulation of the sphere as required. For instance, take an odd number $n>r / 2$ of copies of $R$ and glue them together to form a long hose. Now close this to a sphere by attaching a wheel at each mouth. The middle copy of $\mathbb{R}$ yields the result for $g=0$ by Theorems 2.6 and 3.1.

Observe now that, given two $r$-coaffine triangulations with involutive coaffinations as in (1), we can glue them together to obtain a new $r$-coaffine triangulation: First identify a diamond $\left(=K_{4}-e\right)$ in one of the triangulations with a diamond in the other and remove the diagonal. Now do the same to the coaffine images of the original diamonds. The natural induced involution in the resulting surface triangulation is $r$-coaffine because the distance between symmetric diamonds in each surface is at least $r-2$. Note also that, when both triangulations are orientable and their coaffinations reverse the orientations, the resulting triangulation is also orientable and its coaffination is also orientation-reversing.

Suppose the theorem valid for some orientable surface $\mathcal{S}$ of genus $g$ and fix some $r \geq 3$. Take any triangulation $\mathbb{T}$ of $\mathcal{S}$ as in the statement and any of the above constructed triangulations $\mathbb{S}$ of the sphere. Apply the preceding construction to $\mathbb{T}$ and $\mathbb{S}$, just taking care that the diagonals to be removed in $S$ do not lie in the central copy of $\mathbb{R}$. The resulting triangulation $\mathbb{T}^{\prime}$ still admissibly contains $\mathbb{R}$, so it is rank divergent. Clearly $\mathbb{T}^{\prime}$ has genus $g+1$. 
For the non-orientable case, we shall use some concepts and results form [4]: Let $A$ and $B$ be graphs. A triangular covering map from $A$ to $B$ is any local isomorphism $p: A \rightarrow B$, i.e. a function $p: V(A) \rightarrow V(B)$ such that the restriction $p_{\mid}: N[a] \rightarrow N[p(a)]$ is a graph isomorphism for all $a \in A$. Here $N[a]$ is the closed neighbourhood. In particular, if one of $A$ and $B$ is a Whitney triangulation so is the other. All fibers $p^{-1}(b)$ have the same cardinality: the number of sheets of $p$. Galois triangular covers constitute an important particular case: If $\Gamma$ is a group of automorphisms of $A$ and $d(a, \gamma(a)) \geq 4$ holds for every $a \in A$ and $\gamma \in \Gamma \backslash\{1\}$, we can take the quotient graph $A / \Gamma$ and then the natural projection $p: A \rightarrow A / \Gamma$ is a triangular covering map ([4], Lemma 3.1). Note that every non-trivial element in

$\Gamma$ is a 4-coaffination of $A$. We will also use Proposition 2.2 from [4]:

Proposition 4.2. If $p: A \rightarrow B$ is a triangular covering map, $p_{K}: K(A) \rightarrow K(B)$ is also a triangular covering map with the same number of sheets as $p$. In particular, if $p$ is finite-to-one, $A$ and $B$ have the same $K$-behaviour.

Theorem 4.3. Each closed surface admits infinitely many clique divergent Whitney triangulations.

Proof. For orientable surfaces we use 4.1. Fix a non-orientable closed surface $\mathcal{S}$, and call $\chi=\chi(\mathcal{S})$ its Euler characteristic. Let $\mathcal{T}$ be the orientable closed surface with $\chi(\mathcal{T})=2 \chi$. Fix $r \geq 4$ and take any triangulation $\mathbb{T}=(T, \sigma)$ of $\mathcal{T}$ as in 4.1. Putting $\Gamma=\{1, \sigma\}$, consider $S=T / \Gamma$ and the Galois cover $p: T \rightarrow S$. Then $S$ is a Whitney triangulation of some surface $\mathcal{S}^{\prime}$, and $\chi\left(\mathcal{S}^{\prime}\right)=\chi$ because $p$ is two-sheeted. Since $\sigma$ is orientation-reversing, $\mathcal{S}^{\prime}$ is non-orientable, so it is homeomorphic to $\mathcal{S}$. Therefore $S$ is a Whitney triangulation of $\mathcal{S}$, and it is clique divergent by 4.2 .

\section{Open PROBlems}

It was proved in [7] that almost every surface admits a clique convergent Whitney triangulation. The possible exceptions are those with non-negative Euler characteristic: the sphere, the projective plane, the torus and the Klein bottle.

The following problem immediately arises:

Problem 1. [7] Determine if there are clique convergent Whitney triangulations for the sphere, the projective plane, the torus and the Klein bottle.

This is intriguingly connected to an older conjecture: We have a lot of experimental evidence suggesting that the sphere might not have such a triangulation (see for instance [8]).

Conjecture 1. [6] Every Whitney triangulation of the sphere is clique divergent.

However, it seems that just the opposite happens if we simply remove one edge from such a triangulation of the sphere. More generally, we have:

Conjecture 2. [6] Every Whitney triangulation $T$ of the disk is clique null (i.e. $K^{n}(T)$ is the trivial graph for some $n$ ).

Conjecture 1 can be very challenging even for small particular cases. We have not been able to determine the $K$-behaviour of the snub disphenoid depicted in Fig. 4 (this graph is also known as the siamese dodecahedron, the 12-faced convex deltahedron and the $84^{\text {th }}$ Johnson solid: $J_{84}$ ). The orders of its first iterated clique 
graphs are 8, 12, 20,56 and 1076. We know for sure that the $5^{\text {th }}$ iterated clique graph has more than 7.37 billion vertices. An analysis of the binary subtree which was explored during 30 days by the algorithm of Bron and Kerbosch [1] (implemented in GAP [2]) allows us to suspect that the order of this $5^{\text {th }}$ clique graph is likely around $10^{22}$. Determining the $K$-behaviour of this graph will almost certainly require the development of novel techniques.

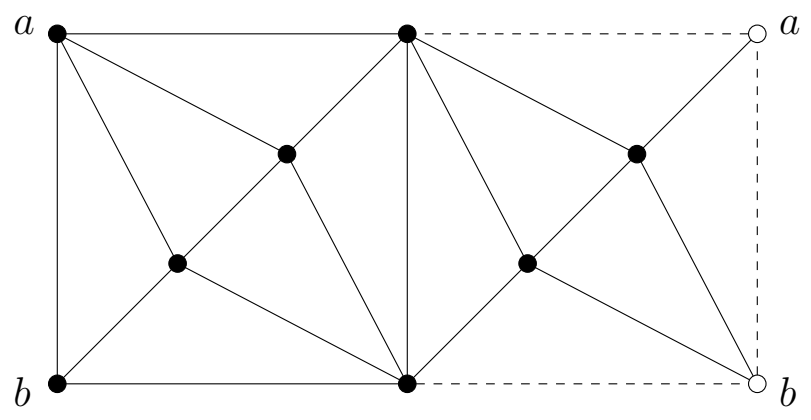

Figure 4. A graph with unknown $K$-behaviour

Note that Conjecture 1 would imply, by Theorem 4.2, that also every Whitney triangulation of the projective plane is clique divergent.

As regards Conjecture 2, we point out that a special case is solved:

Theorem 5.1. [7] Let $G$ be a Whitney triangulation of the disk such that every interior vertex has degree $\geq 6$. Then $G$ is clique null.

\section{REFERENCES}

[1] C. Bron and J. Kerbosch. Finding all cliques of an undirected graph-algorithm 45\%. Communications of the ACM 16 (1973) 575-577.

[2] The GAP Group. GAP - Groups, Algorithms, and Programming, Version 4.3, 2002. (http://www.gap-system.org).

[3] N. Hartsfield and G. Ringel. Clean Triangulations. Combinatorica 11 (1991) 145-155.

[4] F. Larrión and V. Neumann-Lara. Locally $C_{6}$ graphs are clique divergent. Discrete Math. 215 (2000) 159-170.

[5] F. Larrión and V. Neumann-Lara. On clique-divergent graphs with linear growth. Discrete Math. 245 (2002) 139-153.

[6] F. Larrión, V. Neumann-Lara and M.A. Pizaña. Whitney triangulations, local girth and iterated clique graphs. Discrete Math. 258 (2002) 123-135.

[7] F. Larrión, V. Neumann-Lara and M.A. Pizaña. Clique Convergent Surface Triangulations. Matemática Contemporânea 25 (2003) 135-143.

[8] F. Larrión, V. Neumann-Lara and M.A. Pizaña. Clique divergent clockwork graphs and partial orders. Discrete Appl. Math. 141 (2004) 195-207.

[9] V. Neumann-Lara. On clique-divergent graphs, in Problèmes Combinatoires et Théorie des Graphes (Colloques internationaux CNRS, 260) Editions du CNRS, Paris, 1978, 313-315.

[10] V. Neumann-Lara. Clique divergence in graphs, in Algebraic methods in graph theory (Coll. Math. Soc Janos Bolyai, 25, Szeged, 1978) North-Holland, Amsterdam, 1981, 563-569.

[11] M.A. Pizaña. The icosahedron is clique divergent. Discrete Math. 262 (2003) 229-239.

[12] E. Prisner. Graph dynamics. Longman, Harlow, 1995.

[13] W.T. Tutte. A census of plane triangulations. Canad. J. Math. 14 (1962), 21-28.

[14] H. Whitney. A Theorem on Graphs. Ann. Math. 32 (1931) 378-390.

[15] J.L. Szwarcfiter. A survey on clique graphs, in Recent Advances in Algorithms and Combinatorics. B.A. Reed and C.L. Sales, eds. (CMS Books in Mathematics/Ouvrages de Mathématiques de la SMC, 11). Springer-Verlag, New York (2003) 109-136. 
F. Larrión.paco@math.unam.mx

Instituto de Matemáticas, U.N.A.M.

Circuito Exterior, C.U. México 04510 D.F. MEXICO.

V. Neumann-Lara. neumann@math.unam.mx

Instituto de Matemáticas, U.N.A.M.

Circuito Exterior, C.U. México 04510 D.F. MEXICO.

M.A. Pizaña. map@xanum.uam.mx

http://xamanek.izt.uam.mx/map.

Universidad Autónoma Metropolitana,

Depto. de Ingeniería Eléctrica.

Av. San Rafael Atlixco 186, Col Vicentina, México 09340 D.F. MEXICO. 